\title{
Vibrational Dynamics of Phenylphenanthrenes with Phenyl Group at Different Positions
}

\author{
Rashmi Singh ${ }^{1}$, Shantanu Rastogi ${ }^{2} *$ \\ ${ }^{1,2}$ Department of Physics, D.D.U. Gorakhpur University, Gorakhpur, India - 273009 \\ *Corresponding Author: shantanu_r@hotmail.com, Tel.: +91-9335631805
}

Available online at: www.isroset.org

Received: 22/Sept/2018, Accepted: 10/Oct/2018, Online: 31/Oct/2018

\begin{abstract}
Polycyclic aromatic hydrocarbons (PAHs) appear to be ubiquitous in terrestrial atmosphere as well as in the interstellar medium (ISM). In the astrophysical context presence of PAHs is interpreted by the observations of mid-IR emission bands at 3.3, 6.2, 7.7, 8.6, 11.2 and 12.6 micron $\left(3030,1610,1300,1160,890\right.$ and $\left.790 \mathrm{~cm}^{-1}\right)$. The profile of these emission bands exhibit definite variations from source to source, which are explained due to sources having different types of PAHs in different ionization states. The model spectra of mixtures of PAHs show good fit for most bands except for the 6.2 micron feature. Thus a wider variety of PAHs need to be considered for this feature. Therefore, in the present work phenyl substituted phenanthrenes are studied. PAH formation in circumstellar medium of planetary nebulae and dense clouds do indicate formation of phenyl radical and phenyl substituted PAHs. To understand the modifications in infrared lineaments upon phenyl substitution and also due to substitution at different positions, study is made on the five isomers of phenylphenanthrene. The features that may account for possible phenylphenanthrenes in protoplanetary nebulae atmospheres are discussed. Possible contribution of phenylphenanthrenes towards the 6.2 micron feature and other astrophysical emission bands is assessed.
\end{abstract}

Keywords-PAH, Aromatic Infrared Bands, Interstellar molecules, DFT calculations, Astrochemistry

\section{INTRODUCTION}

Among the carbonaceous molecules polycyclic aromatic hydrocarbons (PAHs) are ubiquitously found in terrestrial and in extraterrestrial space. In the Earth's atmosphere PAHs get introduced via any incomplete combustion process [1]. In space the circumstellar shells of carbon rich stars in late stages of evolution have similar but low-density combustion like atmospheres [2]. PAH formation in planetary nebulae environments are envisaged through a bottom-up reaction approach [3]. Being very stable molecules, the PAHs seem to survive in harsh astrophysical conditions and are also assumed to form through defragmentation or surface etching of grains in a top-down approach [4]. On Earth PAHs are well identified, several of these being carcinogenic and health hazard [5]. Whereas, in space PAHs are interpreted on the basis of their infrared emission bands with no specific PAH identification possible.

Astrophysical infrared PAH emission bands, referred to as aromatic infrared bands (AIBs), appear at 3.3, 6.2, 7.7, 8.6, 11.2 and $12.6 \mu \mathrm{m}(3030,1610,1300,1160,890$ and 790 $\mathrm{cm}^{-1}$ ) [6-9]. These features result from mixing of emissions from a population of PAHs possible in that environment. The variations in these features are correlated with the type of astrophysical object and thus indicate different types of PAHs in different environments [10]. Study of infrared spectroscopic properties of different types of PAHs in different ionization states is, therefore, important to decipher astrophysical observations. This in turn helps in improving understanding of the physical and chemical evolution of various objects. 


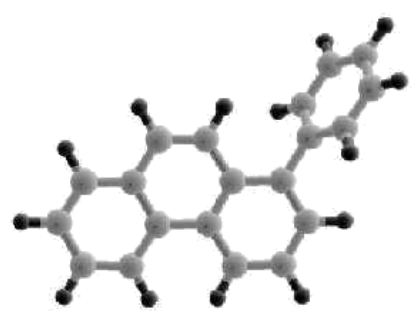

1-phenvlphenanthrene:
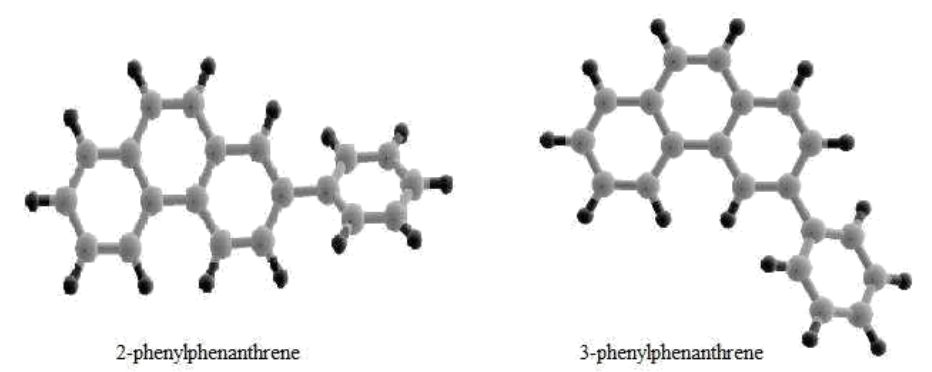

3 phenylphenanthrene

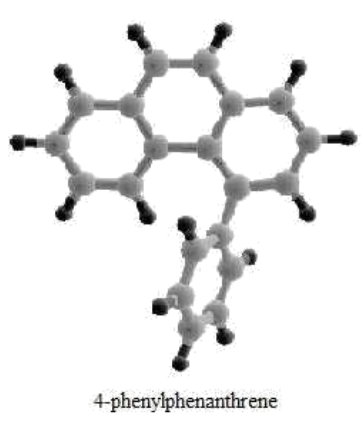

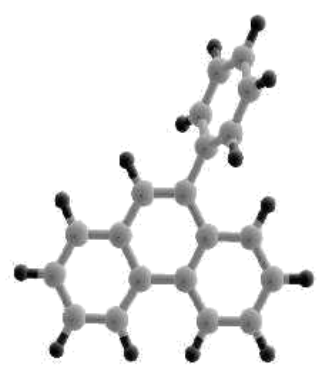

9-phenylphenanthrene

Figure 1 Molecular structures of five phenylphenanthrene isomers.

The astrophysical PAH formation scenario indicates phenyl radical as an important intermediate and may play a major role in chemistry of the interstellar medium (ISM) [11]. Modelling protoplanetary nebula conditions, of dense-warm gas irradiated by a strong UV field, has shown to produce benzene [12]. Laboratory study of shock against benzene yield phenanthrene and phenylphenanthrenes [13]. Similar conditions are there in the expanding envelopes of protoplanetary nebulae, suggesting phenylphenanthrenes in the ISM. The presence of phenyl as a side group in aliphatic compounds and in complex systems show a medium intense mode close to the $6.2 \mu \mathrm{m}$ AIB $[14,15]$. This AIB remains unexplained by considering plain PAHs [16 - 19]. In this communication vibrational dynamics study of all five possible isomers of phenylphenanthrenes, shown in Figure 1, is performed. The paper is organized as follows, Section I gives the background information of the problem and covers the motivation behind the current work, Section II details the theoretical methods and results of computation, Section III presents vibrational analysis to discuss the modifications in the infrared features due to different attachment position of phenyl group and due to ionization, Section IV presents the astrophysical aspects and the possibility of incorporation of phenylphenanthrenes in explaining astrophysical bands and Section V provides the concluding highlights the work and gives pointers for possible future study.

\section{Theoretical Computation}

The type of PAHs that are possible in diverse conditions of the ISM may not all be available terrestrially for laboratory study. Quantum chemical calculations provide a useful tool to simulate infrared spectrum of such PAHs and consider their contribution towards AIBs [10,16-21]. Even where the sample $\mathrm{PAH}$ is available, correct spectra of isolated molecule, as is possible in the ISM, is difficult in the laboratory. Theoretical computations are capable of providing suitable information for such systems.

As phenanthrene and phenylphenanthrenes are possible in shock regions [13] it is important to study their vibrational modes. In laboratory the four isomers 1-, 2-, 3- and 4phenylphenanthrene are obtained by photocyclization of phenylstilbenes [22], while 9-phenylphenanthrene is obtainable via photocatalytic benzannulation [23]. The vibrational spectroscopic features of phenylphenanthrenes are not available in literature so a theoretical study of their infrared spectra is performed. The GAMESS ab initio program [24] is used to obtain optimized molecular structure and infrared vibrational frequencies. The calculations are performed using density functional theory (DFT) with B3LYP functionals in combination with the 6-31G(d) basis set. Study is performed on both neutrals and cations as molecules in the ISM are likely to be also in ionized form.

The phenyl moiety is out-of-plane with respect to the phenanthrene unit due to the steric hinderance from nearby hydrogens. Both the structure and stability of phenylphenanthrene isomers depend on the hydrogen placement with respect to the attached phenyl ring. The stability can be interpreted on the basis of the sharpness of minimized energy with respect to the out-of-plane dihedral angles of the phenyl moiety. The plot of energy vs. angle of 
phenyl torsion are shown in Figure 2a for 1-, 4- and 9phenylphenanthrene, where the minima is at $\sim 55^{\circ}$ and there is little increase in energy beyond the minima. For 2- and 3phenylphenanthrene, shown in Figure $2 b$, the torsion angle minima are at $\sim 40^{\circ}$. The environment of the phenyl group is similar in 2- and 3-phenylphenanthrene with symmetric placement of hydrogen atoms. The placement of hydrogens in 1-, 4- and 9-phenylphenanthrene is not symmetric (Figure 1).

The final optimized phenyl torsion angles are given in Table 1 along with the computed energies for both neutrals and cations. Upon ionization the torsion angle is reduced in all the isomers. The structural similarity of $2-$ and $3-$ phenylphenanthrene, with respect to hydrogens surrounding the phenyl group, is reflected in their nearly equal minimized energies. These two conformers also have lowest energy indicating that symmetric hydrogen placement brings more stability to these isomers. The study of distribution and occurrence of phenylated aromatics in geological samples shows the presence of phenylphenanthrenes $[25,26]$ wherein 2 - and 3-phenylphenanthrene are more prevalent than other isomers [26]. The highest optimized energy is that of 4phenylphenanthrene, which is the most sterically hindered isomer having phenyl group in the bay region of phenanthrene.

\begin{tabular}{|l|c|c|c|c|c|}
\hline Table 1. Optimized geometry-phenyl group torsion angle and energies \\
\hline Phenylphenanthrenes & $\begin{array}{c}\text { torsion angle } \\
\text { (degree) }\end{array}$ & \multicolumn{2}{c|}{$\begin{array}{c}\text { optimization energy } \\
\text { (Kcal/mol) } \\
\mathrm{E}\end{array}$} & $\begin{array}{c}\text { Energy } \\
\text { change } \\
\Delta \mathrm{E}\end{array}$ \\
\hline & neutral & cation & Neutral & cation & \\
\hline 1-Phenylphenanthrene & 56.2 & 43.2 & -770.09704 & -769.84341 & -0.2536 \\
\hline 2-Phenylphenanthrene & 36.9 & 23.6 & -770.10194 & -769.84511 & -0.2568 \\
\hline 3-Phenylphenanthrene & 41.1 & 24.2 & -770.10185 & -769.84512 & -0.2567 \\
\hline 4-Phenylphenanthrene & 61.3 & 43.3 & -770.08865 & -769.83260 & -0.2561 \\
\hline 9-Phenylphenanthrene & 56.3 & 42.6 & -770.09736 & -769.84515 & -0.2522 \\
\hline
\end{tabular}

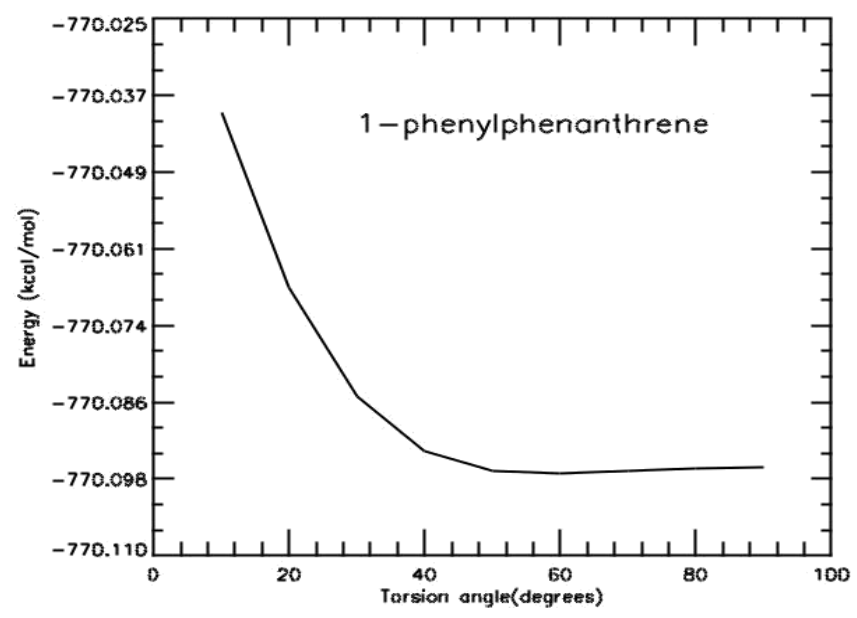

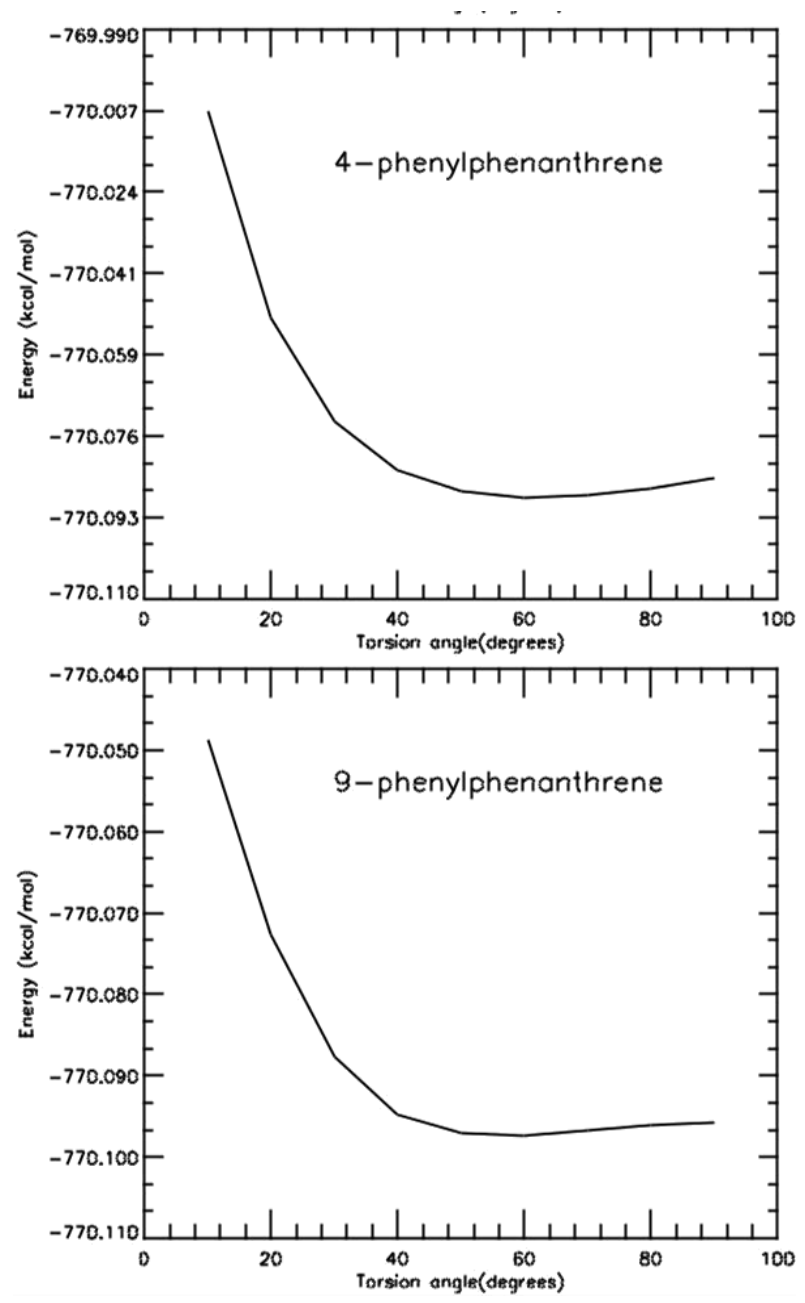

Figure 2a. Variation of optimization energy with torsion angle of the phenyl group substituted in asymmetric $\mathrm{H}$ position.

The vibrational modes are computed using the optimized geometry. The frequencies obtained are usually overestimated and need to be scaled. The scaling procedure and factors depend upon the level of theory and basis sets [27]. The 6-31G(d) basis set is used in the current computations for phenylphenanthrenes and the two level frequency scaling [27] is applied. The scale factor for $\mathrm{C}-\mathrm{H}$ stretching vibrations computed around $3000 \mathrm{~cm}^{-1}$ is 0.9603 and that for the $1800-500 \mathrm{~cm}^{-1}$ frequency range it is 0.9697 . 

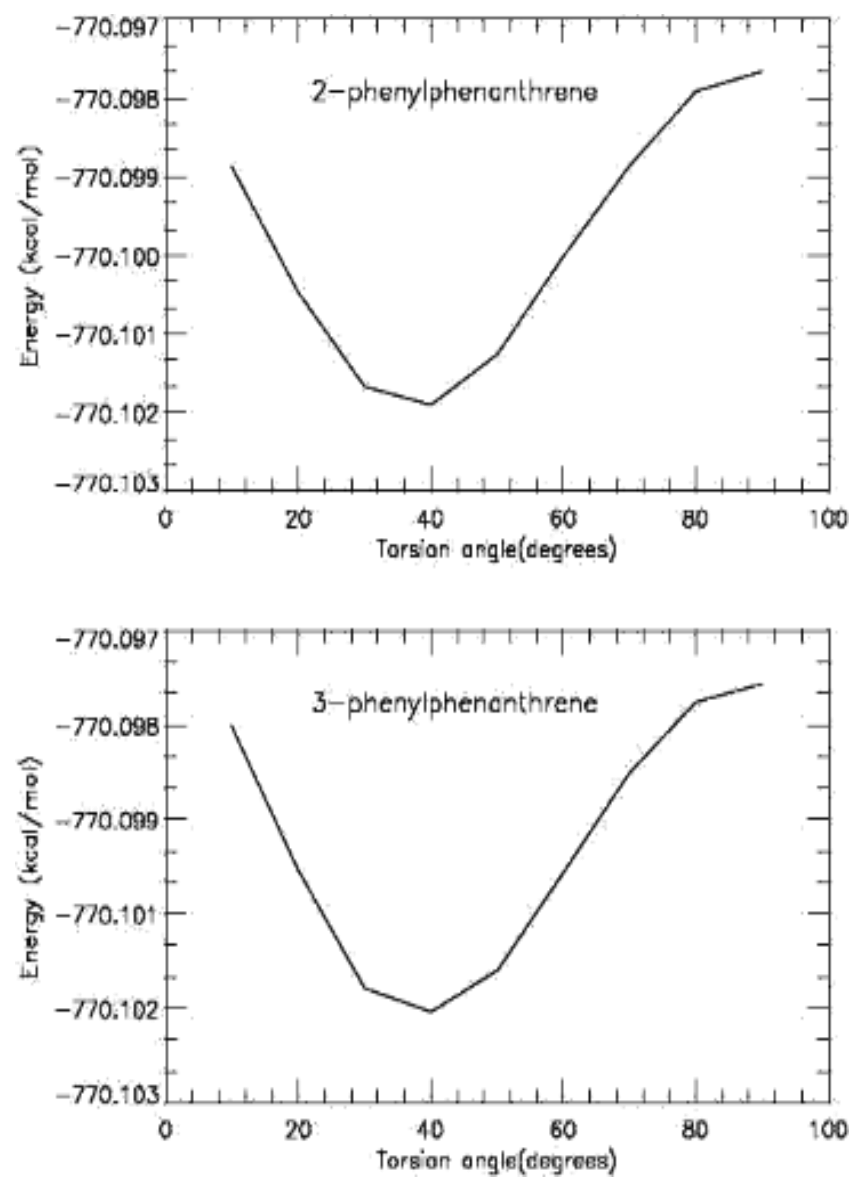

Figure $2 b$. Variation of optimization energy with torsion angle of the phenyl group substituted in symmetric $\mathrm{H}$ position.

\section{VibRational ANALYSIS}

The scaled frequencies and the corresponding infrared intensities are used to simulate absorption spectrum of each isomer. The peaks are considered having Lorentzian profile with $5 \mathrm{~cm}^{-1}$ FWHM and the intensities are taken relative to the most intense mode. These spectra are shown in Figures 3 -7 for the frequency range $1800-500 \mathrm{~cm}^{-1}$, wherein vertical dotted lines show the position of 6.2 and $7.7 \mu \mathrm{m}$ AIB. The C$\mathrm{H}$ stretch mode frequencies, around $3000 \mathrm{~cm}^{-1}$, fall close together and combine into a complex feature as shown in Figure 8. The frequencies and corresponding infrared intensities for each molecule and its cation are given in Appendix Tables 2-6, where only the modes having relative intensity $\geq 0.01$ are given. Just like in plain PAHs [16 - 18] neutral phenylphenanthrenes also have intense $\mathrm{C}-\mathrm{H}$ stretch mode peaks that fall to very low intensities upon ionization. Ionization also leads to strong peaks in the $1600-1100 \mathrm{~cm}^{-1}$ region corresponding to $\mathrm{C}-\mathrm{C}$ stretch and in-plane bend modes.
In neutral phenylphenanthrenes prominent bands are related to motion of hydrogens, i.e. $\mathrm{C}-\mathrm{H}$ stretching vibration in the $3000 \mathrm{~cm}^{-1}$ region and C-H out-of-plane wag motion in $600-$ $900 \mathrm{~cm}^{-1}$ range. Although the hydrogens are attached to similar aromatic carbons yet even slight difference in nonbonded environment may change the vibrational frequency and intensity of the modes. In PAHs the hydrogen motions can be different according to it being attached to a ring that has quarto (four), trio (three), duo (two) or solo (one) peripheral hydrogens $[28,29]$. In addition to a duo and two quarto hydrogens, there is also a bay region in phenanthrene [30], which when substituted with a phenyl group forms 4 phenylphenanthrene. Important modes in the five isomers are shown in Table 7. The features of phenanthrene spectrum [17] are only slightly disturbed by the addition of phenyl group. The phenyl group substitution induces its own bands and as the symmetry of phenanthrene is broken a few more modes get active. The phenyl group $\mathrm{C}-\mathrm{H}$ wag is observed at $\sim 695 \mathrm{~cm}^{-1}$ in all isomers with nearly equivalent intensity. The most intense single quarto $\mathrm{C}-\mathrm{H}$ wag peak of phenanthrene [17] at $\sim 740 \mathrm{~cm}^{-1}$ is two peaks in phenylphenanthrenes. In 1-phenylphenanthrene these are very close peaks of nearly equal strength, arising due to the breaking of phenanthrene symmetry on phenyl addition. In the other isomers the two peaks are about $20 \mathrm{~cm}^{-1}$ apart with the lower frequency quarto $\mathrm{C}-\mathrm{H}$ wag mode being stronger. The higher frequency mode is due to the $\mathrm{C}-\mathrm{H}$ wag in the phenyl group. Another strong $\mathrm{C}-\mathrm{H}$ wag mode of phenanthrene at $825 \mathrm{~cm}^{-1}$ [17] is similar in 3- and 4phenylphenanthrene but it appears at $803 \mathrm{~cm}^{-1}$ in $1-$ and $2-$ phenylphenanthrene. In 9-phenylphenanthrene there is altogether a different mode at $769 \mathrm{~cm}^{-1}$.

The features of phenanthrene spectrum [17] are only slightly disturbed by the addition of phenyl group. The phenyl group substitution induces its own bands and as the symmetry of phenanthrene is broken a few more modes get active. The phenyl group $\mathrm{C}-\mathrm{H}$ wag is observed at $\sim 695 \mathrm{~cm}^{-1}$ in all isomers with nearly equivalent intensity. The most intense single quarto C-H wag peak of phenanthrene [17] at 740 $\mathrm{cm}^{-1}$ is two peaks in phenylphenanthrenes. In $1-$ phenylphenanthrene these are very close peaks of nearly equal strength, arising due to the breaking of phenanthrene symmetry on phenyl addition. In the other isomers the two peaks are about $20 \mathrm{~cm}^{-1}$ apart with the lower frequency quarto $\mathrm{C}-\mathrm{H}$ wag mode being stronger. The higher frequency mode is due to the $\mathrm{C}-\mathrm{H}$ wag in the phenyl group. Another strong $\mathrm{C}-\mathrm{H}$ wag mode of phenanthrene at $825 \mathrm{~cm}^{-1}$ [17] is similar in 3- and 4-phenylphenanthrene but it appears at 803 $\mathrm{cm}^{-1}$ in 1- and 2-phenylphenanthrene. In 9phenylphenanthrene there is altogether a different mode at $769 \mathrm{~cm}^{-1}$.

The strongest features in the neutral molecules are due to C$\mathrm{H}$ stretch modes, the last column in Table 7. For all isomers 
the most intense mode is at around $3070 \mathrm{~cm}^{-1}$, due mainly to the $\mathrm{C}-\mathrm{H}$ stretching in the phenanthrene unit. The strong higher frequency mode at $\sim 3090 \mathrm{~cm}^{-1}$ is due to the $\mathrm{C}-\mathrm{H}$ stretch of the two bay hydrogens. In 4-phenylphenanthrene this mode is absent, possibly as one bay hydrogen is substituted by the phenyl group.
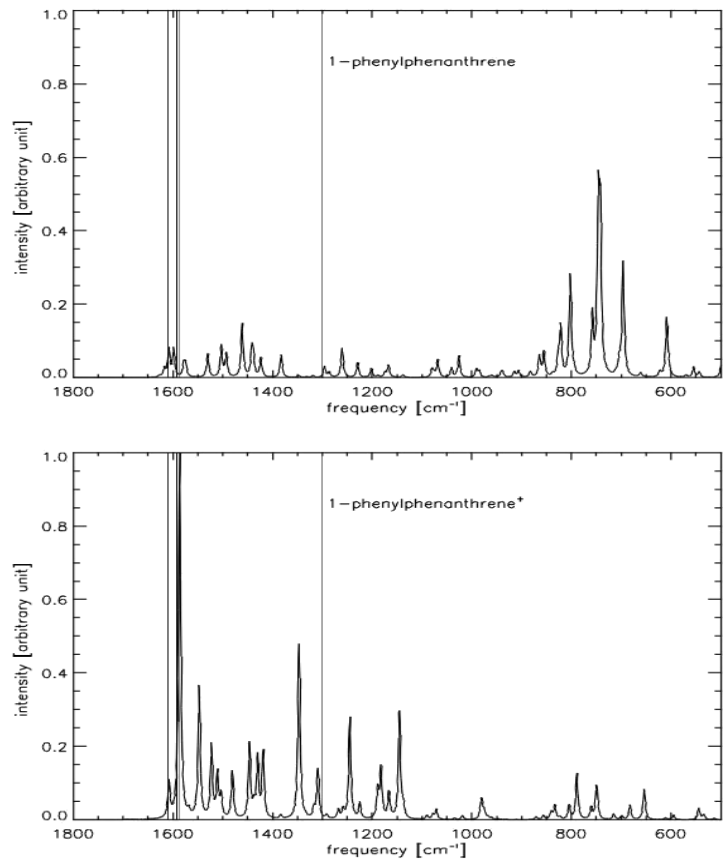

Figure 4. Infrared spectra of 1-phenylphenanthrene neutral and cation.
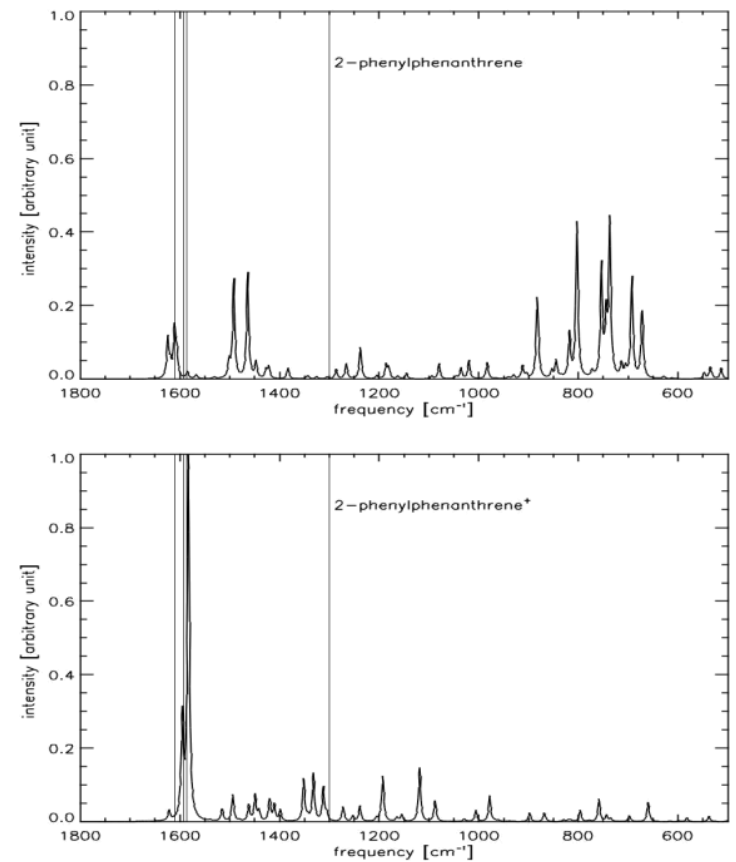

Figure 4. Infrared spectra of 2-phenylphenanthrene neutral and cation.
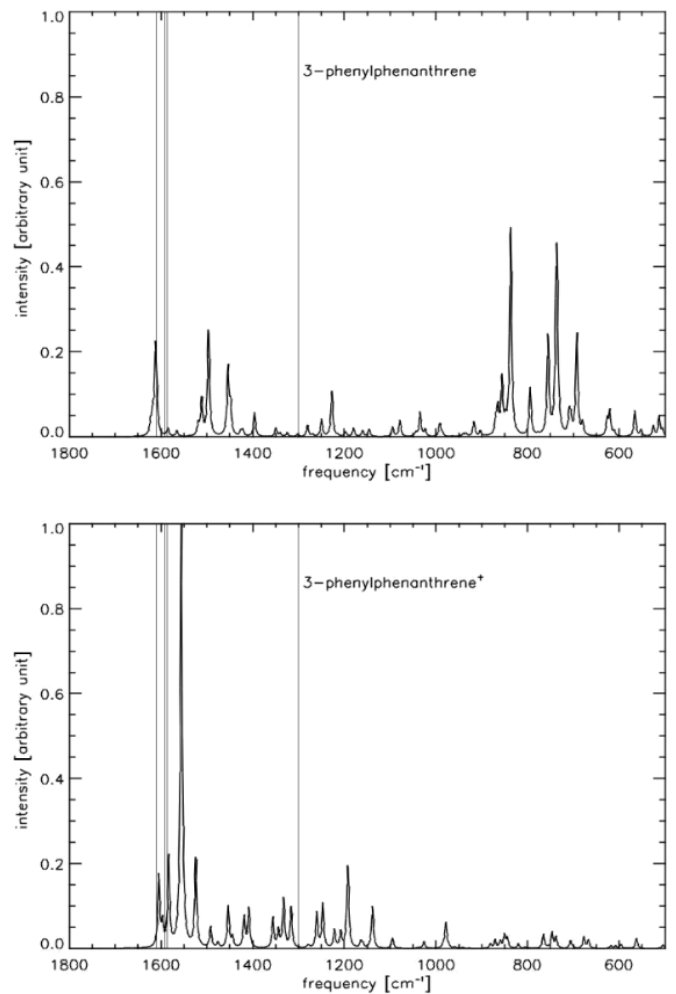

Figure 5. Infrared spectra of 3-phenylphenanthrene neutral and cation.
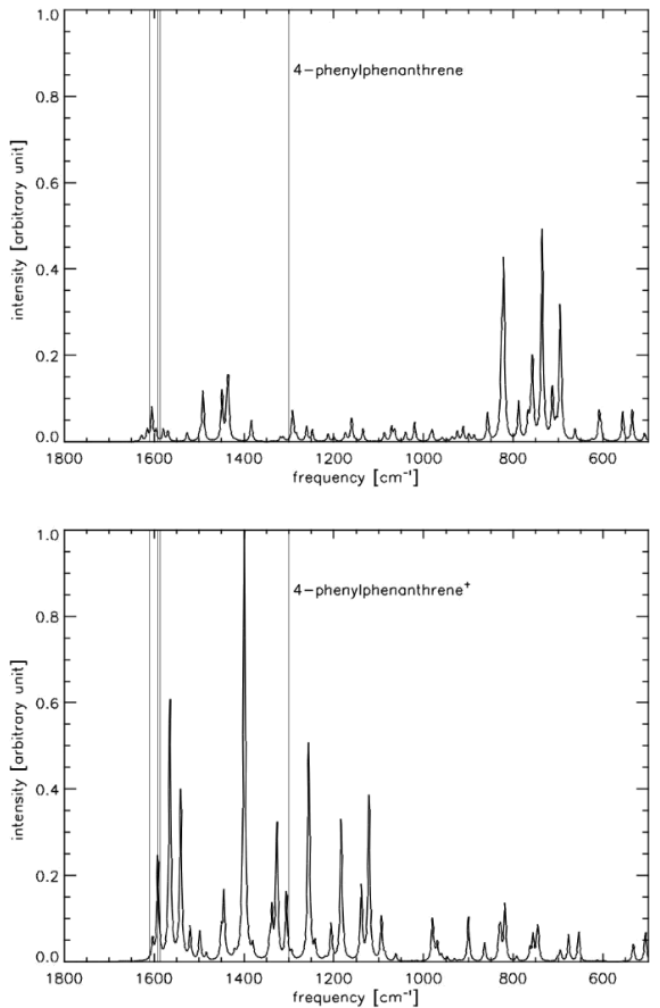

Figure 6. Infrared spectra of 4-phenylphenanthrene neutral and cation. 

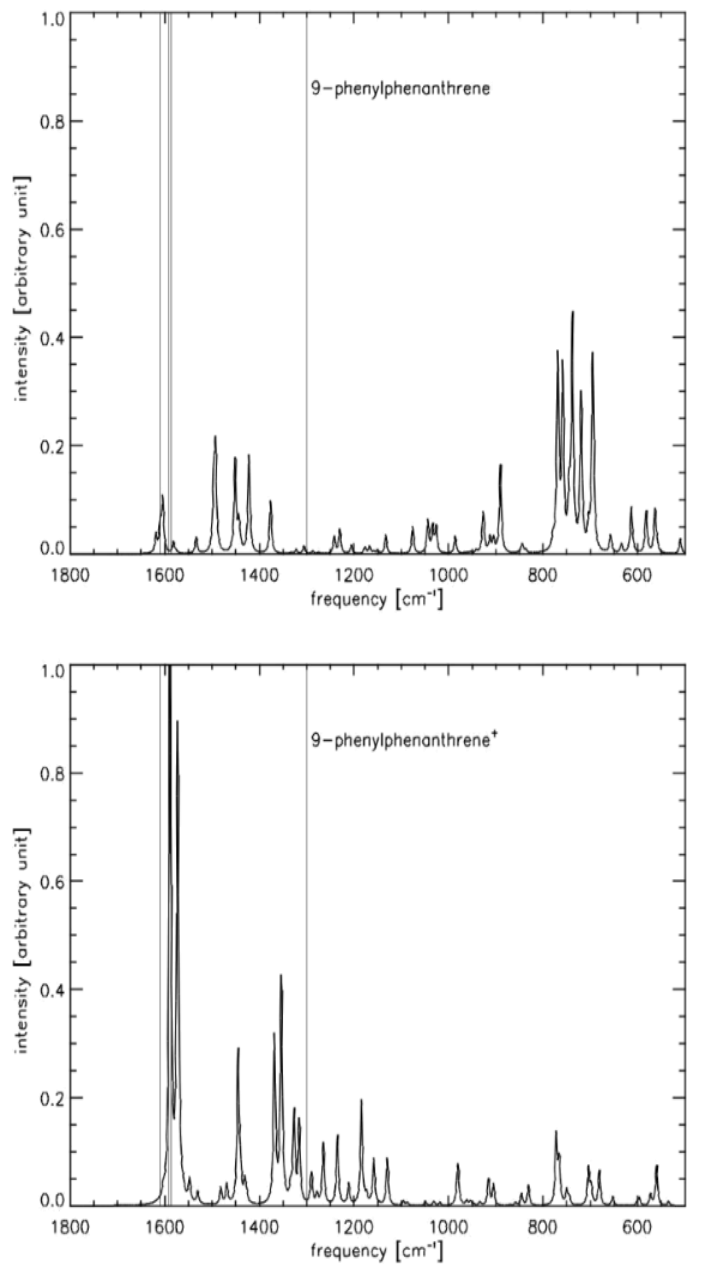

Figure 7. Infrared spectra of 9-phenylphenanthrene neutral and cation.
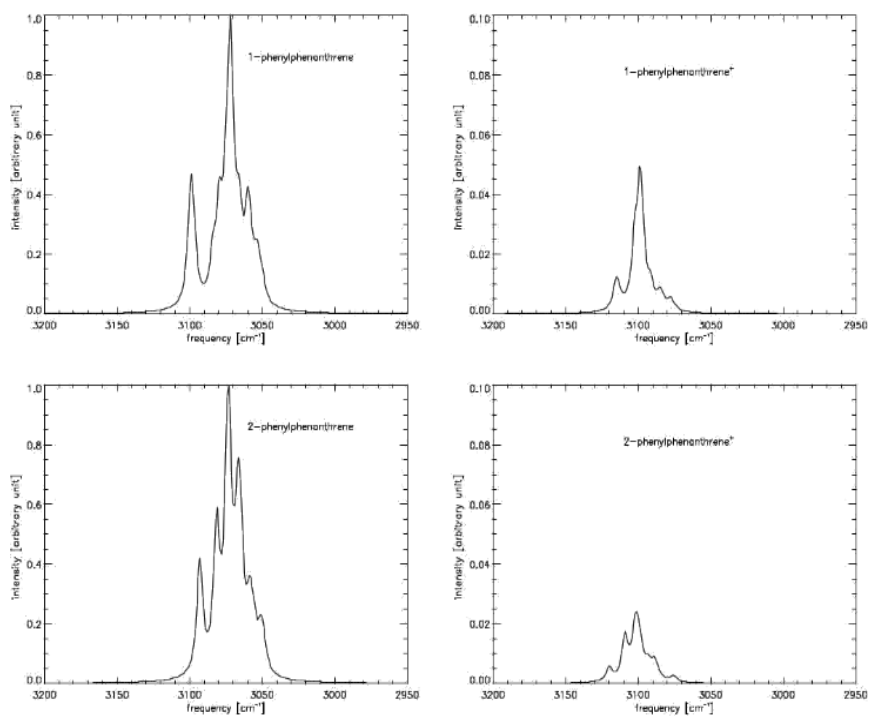
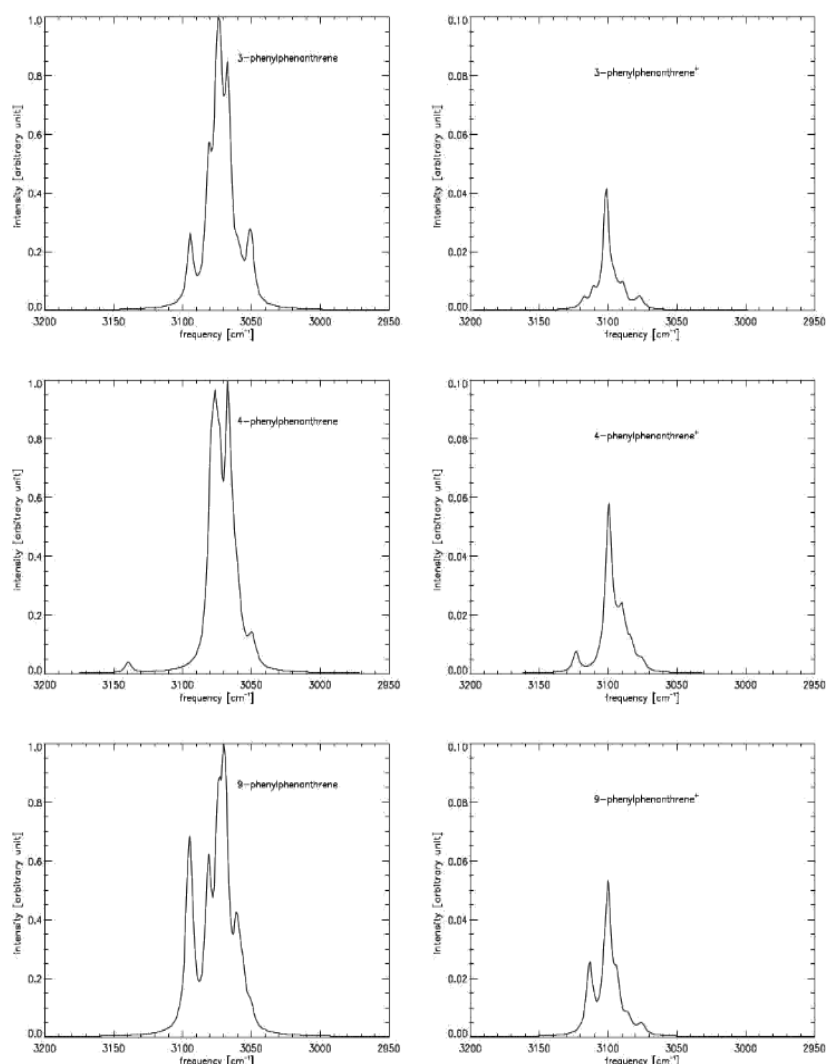

Figure 8. The $\mathrm{C}-\mathrm{H}$ stretch spectral region of infrared spectra in neutrals and cations of phenylphenanthrenes.

Among other significant modes in the neutrals are planar ring deformation modes listed in Table 8. Two peaks at around 1490 and $1455 \mathrm{~cm}^{-1}$ related to C-C stretch and C-H in-plane.

Table 7. Prominent frequencies corresponding to modes involving motion of hydrogens in neutrals.

\begin{tabular}{|c|c|c|c|c|}
\hline phenylphenanthrenes & C-H wag & \multicolumn{2}{|c|}{ C-H wags } & C-H stretch \\
\hline neutral & Phenyl & \multicolumn{2}{|c|}{ phenanthrene unit } & \\
\hline 1-phenylphenanthrene & $\begin{array}{c}697 \\
(0.56)\end{array}$ & $\begin{array}{c}742 \\
(0.70) \\
746 \\
(0.75)\end{array}$ & $\begin{array}{c}803 \\
(0.49)\end{array}$ & $\begin{array}{c}3072(1.0) \\
3099(0.63)\end{array}$ \\
\hline 2-phenylphenanthrene & $\begin{array}{c}693 \\
(0.49)\end{array}$ & $\begin{array}{c}737 \\
(0.76) \\
754 \\
(0.54)\end{array}$ & $\begin{array}{c}803 \\
(0.76)\end{array}$ & $\begin{array}{c}3073(1.0) \\
3081(0.8), 3092 \\
(0.67)\end{array}$ \\
\hline 3-phenylphenanthrene & $\begin{array}{c}693 \\
(0.42)\end{array}$ & $\begin{array}{c}736 \\
(0.64) \\
756 \\
(0.42)\end{array}$ & $\begin{array}{c}837 \\
(0.87)\end{array}$ & $\begin{array}{c}3074(1.0) \\
3081(0.6), 3094(0.4)\end{array}$ \\
\hline 4-phenylphenanthrene & $\begin{array}{l}696 \\
(0.55)\end{array}$ & $\begin{array}{c}736 \\
(0.86) \\
758 \\
(0.32)\end{array}$ & $\begin{array}{c}822 \\
(0.67)\end{array}$ & $\begin{array}{c}3067(1.0), 3076 \\
(0.90) \\
3079(0.67)\end{array}$ \\
\hline 9-phenylphenanthrene & $\begin{array}{c}695 \\
(0.63)\end{array}$ & $\begin{array}{c}738 \\
(0.74) \\
759 \\
(0.56)\end{array}$ & 769 (0.6) & $\begin{array}{c}3070(1.0) \\
3094(0.83)\end{array}$ \\
\hline
\end{tabular}


Table 8. The active planar ring deformation modes in neutral and cations. The values in parenthesis are relative intensities.

\begin{tabular}{|c|c|c|c|}
\hline phenylphenanthrenes & \multicolumn{2}{|c|}{ Neutral } & Cation \\
\hline & C-C stretch + C-H in-plane & C-C stretch & C-C stretch \\
\hline 1-phenylphenanthrene & $1461(0.25), 1503(0.15)$ & $1599(0.13), 1608(0.13)$ & $1587(1.0)$ \\
\hline 2-phenylphenanthrene & $1464(0.51), 1492(0.48)$ & $1606(0.11), 1611(0.23)$ & $1583(1.0)$ \\
\hline 3-phenylphenanthrene & $1454(0.28), 1497(0.45)$ & $1612(0.35)$ & $1556(1.0)$ \\
\hline 4-phenylphenanthrene & $1449(0.20), 1491(0.20)$ & $1605(0.14)$ & $1565(0.59), 1592(0.23)$ \\
\hline 9-phenylphenanthrene & $1451(0,30), 1493(0.30)$ & $1605(0.17)$ & $1589(1.0), 1604(0.17)$ \\
\hline
\end{tabular}

bend modes are common in all isomers. The mode close to $1500 \mathrm{~cm}^{-1}$ is reminiscent of the peak in phenanthrene [17].

In cations the intensity of $\mathrm{C}-\mathrm{H}$ stretch modes is extremely small. The scale is magnified in the right column of Figure 8 to clarify the features. Additionally, the intensity of several modes in the $1600-1100 \mathrm{~cm}^{-1}$ region is enhanced. The most intense feature is around $1590 \mathrm{~cm}^{-1}$, due to $\mathrm{C}-\mathrm{C}$ stretch in the phenyl group of all isomers except 3- and 4-phenylphenanthrene. In 3-phenylphenanthrene the most intense mode appears at $1556 \mathrm{~cm}^{-1}$ and in 4-phenylphenanthrene it is at $1400 \mathrm{~cm}^{-1}$. The $\mathrm{C}$-H out-of-plane wag modes get slightly shifted in the cations, while their absolute intensity is not much changed. Their relative intensity appears to be small as the reference intensity of $\mathrm{C}-\mathrm{C}$ stretch mode is very high.

\section{ASTROPHYSICAL ASPECTS}

Study of shock against benzene in laboratory has shown to yield phenanthrene and phenylphenanthrenes [13]. Similar conditions are there in the expanding envelopes of protoplanetary nebulae, suggesting possibility of reactions leading to growth of phenylphenanthrenes in the ISM. The identification of phenylphenanthrenes in geological samples from different eras $[25,26]$ also indicate the ease of growth for phenylated PAHs in carbonaceous environments. Although the astrophysical AIBs get explained through emission models using plain unsubstituted PAHs [10, 21], yet features like the $6.2 \mu \mathrm{m}$ band remain unexplained. Phenyl substitution on phenanthrene make some modes active in this frequency range.

In neutral phenylphenanthrenes the $\mathrm{C}-\mathrm{C}$ stretch modes (Table 8) fall right in the position of Class ' $\mathrm{A}$ ' AIB at $6.2 \mu \mathrm{m}(1610$ $\mathrm{cm}^{-1}$ ) [9], but with moderate intensity. The cations of $1-, 2-$ and 9-phenylphenanthrene do have very strong feature at 6.3 $\mu \mathrm{m}\left(1590 \mathrm{~cm}^{-1}\right)$ that may explain the Class ' $\mathrm{C}$ ' AIB feature observed along protoplanetary nebulae type evolved stars [9].
Other prominent features that appear in the cations of phenylphenanthrenes include modes around 1495 and 1455 $\mathrm{cm}^{-1}(6.7$ and $6.9 \mu \mathrm{m})$. These fall close to AIB sub features observed along some objects. A feature at $6.66 \mu \mathrm{m}$ is observed along IRAS 18434-0242 [31] and in H2 and seyfert 2 galaxies [32]. The $6.9 \mu \mathrm{m}$ feature is seen in proto planetary nebulae [33] and in cool objects [34]. Incorporating suitable phenylphenanthrenes in emission modelling for specific objects is strongly indicated. Modelling of the protoplanetary nebulae $7.7 \mu \mathrm{m}$ feature with plain PAH data point towards large PAHs in these objects [10].

\section{CONCLUSION AND Future SCOPE}

All the isomers of phenylphenanthrene in neutral form have a feature close to $6.2 \mu \mathrm{m}$ but with moderate intensity. It is desirable to incorporate them for modelling the emission of specific objects. The possible objects appear to be those with cool atmospheres as around proto planetary nebulae and stars in the late stage of evolution. The Phenyl substituted large PAHs and other polycyclic conjugated hydrocarbons [35] also need to be studied for possible improvement in the models and to give better insight into the physical and chemical conditions around such stars.

\section{REFERENCES}

[1] H. Choi, R. Harrison, H. Komulainen, J. M. D. Saborit, Polycyclic aromatic hydrocarbons, WHO Guidelines for Indoor Air Quality: Selected Pollutants, Geneva, Ch. 6, pp. 1, 2010.

[2] I. Cherchneff, The formation of Polycyclic Aromatic Hydrocarbons in evolved circumstellar environments, EAS Publications Series, Vol. 46, pp. 177-189, 2011.

[3] A. G. G. M. Tielens, Interstellar Polycyclic Aromatic Hydrocarbon Molecules, Annual Review of Astronomy and Astrophysics, Vol. 4, pp. 289-337, 2008.

[4] P. Merino, M. Svec, J. I. Martinez, P. Jelinek, P. Lacovig, M. Dalmiglio, S. Lizzit, Soukiassian, J. Cernicharo, J. A. Martin-Gago, Graphene etching on sic grains as a path to interstellar polycyclic aromatic hydrocarbons formation, Nature Communications, Vol. 5, No. 3054, 2014.

[5] Working group on PAHs, Ambient Air Pollution by Polycyclic Aromatic Hydrocarbons $(P A H)$, Publications of the European Communities, Luxembourg, 2001. 
[6] M. Cohen, A. G. G. M. Tielens, J. Bregman, F. C. Witteborn, D. M. Rank, L.J. D., Allamandola, Wooden, M. Jourdain de Muizon, The infrared emission bands. III - Southern IRAS sources, Astrophyical Journal, Vol. 341, pp. 246-269, 1989.

[7] D. A. Beintema, M. E. van den Ancker, F. J. Molster, L. B. F. M. Waters, A. G. G. M. Tielens, C. Waelkens, T. de Jong, T. de Graauw, K. Justtanont, I. Yamamura, A. Heras, F. Lahuis, A. Salama, The rich spectrum of circumstellar PAHs, Astronomy \& Astrophysics, Vol. 315, pp. L369L372, 1996.

[8] D. Lutz, H. W. W. Spoon, D. Rigopoulou, A. F. M. Moorwood, R. Genzel, The Nature and Evolution of Ultraluminous Infrared Galaxies:A MidInfrared Spectroscopic Survey, The Astrophysical Journal Letters, Vol. 505, No. 2, pp. L103-L107, 1998.

[9] E. Peeters, S. Hony, C. Van Kerckhoven, A. G. G. M. Tielens, L. J. Allamandola, D. M. Hudgins, C. W. Bauschlicher, The rich 6 to 9 vec mu $m$ spectrum of interstellar PAHs, Astronomy \& Astrophysics, Vol. 390, pp. 1089-1113, 2002.

[10] A. Pathak, S. Rastogi, Modeling the interstellar aromatic infrared bands with co-added spectra of PAHs, Astronomy \& Astrophysics, Vol. 485, No. 3, pp. 735-742, 2008.

[11] R. J. McMahon, M. C. McCarthy, C. A. Gottlieb, J. B. Dudek, J. F. Stanton, $\mathrm{P}$. Thaddeus, The radio spectrum of the phenyl radical, The Astrophysical Journal Letters, Vol. 590, No. 1, pp. L61-L64, 2003.

[12] P. M. Woods, T. J. Millar, A. A. Zijlstra, E. Herbst, The synthesis of benzene in the proto-planetary nebula crl 618, The Astrophysical Journal Letters, Vol. 574, No. 2, pp. L167-L170, 2002.

[13] K. Mimura, R. Sugisaki, Shock reactions of carbon-bearing materials and their cosmochemical significance, High-Pressure Shock Compression of Solids V: Shock Chemistry with Applications to Meteorite Impacts, Springer-Verlag, New York, pp. 75-116, 2003.

[14] P. Tandon, V. D. Gupta, O. Prasad, S. Rastogi, S. B. Katti, Heat capacity and vibrational dynamics of poly ( $\beta$ benzyl-l-aspartate), Journal of Polymer Science Part B: Polymer Physics, Vol. 34, Issue. 7, pp. 1213-1228, 1996.

[15] C. Ngaojampa, S. Namuangruk, Y. Surakhot, V. Promarak, S. Jungsuttiwong, N. Kungwan, Influence of phenyl-attached substituents on the vibrational and electronic spectra of meso-tetraphenylporphyrin: A dft study, Computational and Theoretical Chemistry, Vol. 1062, pp. 1-10, 2015.

[16] S. R. Langhoff, Theoretical infrared spectra for polycyclic aromatic hydrocarbon neutrals, cations, and anions, The Journal of Physical Chemistry, Vol. 100, Issue. 8, pp. 2819-2841, 1996.

[17] A. Pathak, S. Rastogi, Computational study of neutral and cationic catacondensed polycyclic aromatic hydrocarbons, Chemical Physics, Vol. 313, Issue. 1-3, pp. $133-150,2005$.

[18] A. Pathak, S. Rastogi, Computational study of neutral and cationic pericondensed polycyclic aromatic hydrocarbons, Chemical Physics, Vol. 326, pp. 315-328, 2006.

[19] A. Pathak, S. Rastogi, Theoretical infrared spectra of large polycyclic aromatic hydrocarbons, Spectrochimica Acta Part A: Molecular and Biomolecular Spectroscopy, Vol. 67, Issue. 3-4, pp. 898 - 909, 2007.

[20] C. W. Bauschlicher, Jr., E. Peeters, L. J. Allamandola, The Infrared Spectra of Very Large, Compact, Highly Symmetric, Polycyclic Aromatic Hydrocarbons (PAHs), Astrophyical Journal, Vol. 678, pp. 316-327, 2008.

[21] M. J. F. Rosenberg, O. Bern'e, C. Boersma, Random mixtures of polycyclic aromatic hydrocarbon spectra match interstellar infrared emission, Astronomy \& Astrophysics, Vol. 566, No. L4, pp. 1-7, 2014.

[22] S. C. Dickerman, I. Zimmerman, Synthesis of 1-, 2-, 3-, and 4phenylphenanthrenes by photocyclization of isomeric phenylstilbenes, The Journal of Organic Chemistry, Vol. 39, Issue. 23, pp. 3429-3430, 1974.

[23] T. Chatterjee, D. S. Lee, E. J. Cho, Extended study of visible-light-induced photocatalytic [4 + 2] benzannulation: Synthesis of polycyclic (hetero) aromatics, The Journal of Organic Chemistry Vol. 82, Issue. 8, pp. 43694378, 2017.

[24] M. W. Schmidt, K. K. Baldridge, J. A. Boatz, S. T. Elbert, M. S. Gordon, J. H. Jensen, S. Koseki, N. Matsunaga, K. A. Nguyen, S. Su, T. L. Windus,
M. Dupuis, J. A. Montgomery, General atomic and molecular electronic structure system (GAMESS), Journal of Computational Chemistry, Vol. 14, Issue. 11, pp. 1347-1363, 1993.

[25] M. J. Rospondek, L. Marynowski, A. Chachaj, M. Gra, Novel aryl polycyclic aromatic hydrocarbons: Phenylphenanthrene and phenylanthracene identification, occurrence and distribution in sedimentary rocks, Organic Geochemistry, Vol. 40, Issue. 9, pp. 986-1004, 2009

[26] M. Li, S. Shi, T.-G. Wang, N. Zhong, G. Wang, J. Cui, The occurrence and distribution of phenylphenanthrenes, phenylanthracenes and binaphthyls in palaeozoic to cenozoic shales from china, Applied Geochemistry, Vol. 27, Issue. 12, pp. 2560-2569, 2012.

[27] A. Maurya, S. Rastogi, G. Rouill'e, F. Huisken, T. Henning, Experimental and Theoretical Study on the Infrared Spectroscopy of Astrophysically Relevant Polycyclic Aromatic Hydrocarbon Derivatives 2- and 9Vinylanthracene, The Astrophysical. Journal, Vol. 755, No. 2, pp. 1-12, 2012

[28] L. Bellamy, The infrared spectra of complex molecules: advances in infrared group frequencies. vol. two, The Infrared Spectra of Complex Molecules, Chapman and Hall Limited, London, 1980.

[29] D. M. Hudgins, L. J. Allamandola, Interstellar pah emission in the 11-14 micron region: New insightsfrom laboratory data and a tracer of ionized pahs, The Astrophysical Journal Letters, Vol. 516, Issue. 1, pp. L41-L44, 1999.

[30] E. Maltseva, A. Petrignani, A. Candian, C. J. Mackie, X. Huang, T. J. Lee, A. G. G. M. Tielens, J. Oomens, W. J. Buma, High-resolution ir absorption spectroscopy of polycyclic aromatic hydrocarbons in the $3 \mu \mathrm{m}$ region: Role of periphery, The Astrophysical Journal, Vol. 831, No. 1, pp. 1-11, 2016.

[31] E. Peeters, A. G. G. M. Tielens, P. R. Roelfsema, P. Cox, PAHs in compact HII regions, The Universe as Seen by ISO, ESA Special Publication, Vol. 427, pp. 739-741, 1999.

[32] R. J. Laureijs, D. Watson, L. Metcalfe, B. McBreen, B. O'Halloran, J. Clavel, K. Leech, P. Gallais, P. Barr, M. Delaney, L. Hanlon, F. Quilligan, Iso observations of a sample of $60 \mu \mathrm{m}$ peaker galaxies, Astronomy \& Astrophysics, Vol. 359, Issue. 3, pp. 900-906, 2000.

[33] B. J. Hrivnak, K. Volk, S. Kwok, 2-45 Micron Infrared Spectroscopy of Carbon-rich Proto-Planetary Nebulae, The Astrophysical Journal, Vol. 535, pp. 275-292, 2000.

[34] R. H. Buss, Jr., M. Cohen, A. G. G. M. Tielens, M. W. Werner, J. D. Bregman, F. C. Witteborn, D. Rank, S. A. Sandford, Hydrocarbon emission features in the IR spectra of warm supergiants, The Astrophysical Journal Letters, Vol. 365, pp. L23-L26, 1990.

[35] Rushina Natu, Computing the Energy of Some Chemical Graphs Related to Nanostructures in the Case of Alternate Polycyclic Conjugated Hydrocarbons, International Journal of Scientific Research in Physics and Applied Sciences, Vol.3, Issue.2, pp.1-8, 2015.

\section{AUTHORS PROFILE}

Ms. Rashmi Singh is M.Sc. in Physics and is currently pursuing her Ph.D. after qualifying the university research entrance test. She has published one paper and attended several conferences. Her aim is to understand interstellar medium spectroscopically.

Prof. Shantanu Rastogi is M.Sc. and Ph.D. in Physics. He has published more than 40 research papers and supervised 5 Ph.D. thesis. He has more than 25 years research and teaching experience. His main research work focuses on interstellar medium and infrared properties of late type stars.
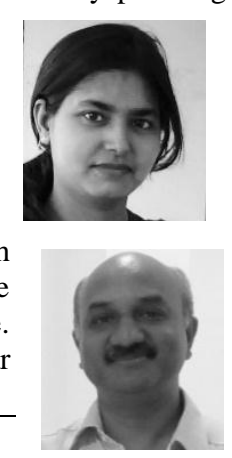


\section{APPENDIX}

Tables 2-6 are presented in this Appendix

Table 2: Infrared frequencies $\left(\mathrm{cm}^{-1}\right)$ and relative intensities for

\begin{tabular}{|c|c|c|c|}
\hline \multicolumn{4}{|c|}{ 1-phenylphenanthrene } \\
\hline & Neutral & & Cation \\
\hline Frequency & Relative Intensity & Frequency & Relative Intensity \\
\hline 99.91 & 0.01 & 118.68 & 0.01 \\
\hline 216.07 & 0.03 & 209.57 & 0.01 \\
\hline 218.17 & 0.01 & 214.67 & 0.01 \\
\hline 300.14 & 0.02 & 245.77 & 0.03 \\
\hline 327.68 & 0.01 & 402.45 & 0.04 \\
\hline 401.16 & 0.02 & 440.5 & 0.01 \\
\hline 407.2 & 0.01 & 465.87 & 0.02 \\
\hline 412.65 & 0.02 & 534.4 & 0.01 \\
\hline 446.88 & 0.08 & 544.43 & 0.03 \\
\hline 490.21 & 0.02 & 595.2 & 0.01 \\
\hline 496.9 & 0.08 & 654.18 & 0.08 \\
\hline 500.18 & 0.02 & 682.67 & 0.04 \\
\hline 543.66 & 0.03 & 699.07 & 0.01 \\
\hline 555.06 & 0.05 & 715.36 & 0.02 \\
\hline 570.09 & 0.01 & 749.01 & 0.06 \\
\hline 604.9 & 0.04 & 750.54 & 0.04 \\
\hline 609.17 & 0.27 & 760.58 & 0.03 \\
\hline 622.68 & 0.03 & 789.25 & 0.13 \\
\hline 661.2 & 0.02 & 804.75 & 0.04 \\
\hline 696.5 & 0.56 & 823.17 & 0.01 \\
\hline 704.41 & 0.06 & 833.58 & 0.04 \\
\hline 711.03 & 0.01 & 841.1 & 0.02 \\
\hline 742.2 & 0.70 & 856.77 & 0.01 \\
\hline 746.22 & 0.75 & 871.22 & 0.01 \\
\hline 758.26 & 0.28 & 967.75 & 0.01 \\
\hline 794.41 & 0.02 & 975.15 & 0.02 \\
\hline 802.62 & 0.49 & 980.35 & 0.05 \\
\hline 821.83 & 0.23 & 983.08 & 0.01 \\
\hline 826.73 & 0.08 & 1018.96 & 0.01 \\
\hline 838.1 & 0.01 & 1071.37 & 0.03 \\
\hline 855.59 & 0.12 & 1078.28 & 0.01 \\
\hline 864.37 & 0.10 & 1090.29 & 0.01 \\
\hline 882.79 & 0.03 & 1137.58 & 0.03 \\
\hline 906.42 & 0.03 & 1145.46 & 0.30 \\
\hline 914.77 & 0.03 & 1166.61 & 0.07 \\
\hline 938.14 & 0.02 & 1182.51 & 0.14 \\
\hline 940.95 & 0.02 & 1189.15 & 0.08 \\
\hline 959.99 & 0.01 & 1225.1 & 0.04 \\
\hline 985.14 & 0.03 & 1245.09 & 0.28 \\
\hline 990.95 & 0.04 & 1258.47 & 0.03 \\
\hline 1026.14 & 0.10 & 1268.11 & 0.03 \\
\hline 1040.98 & 0.05 & 1291.59 & 0.01 \\
\hline 1068.51 & 0.08 & 1309.36 & 0.14 \\
\hline 1076.18 & 0.02 & 1317.35 & 0.03 \\
\hline 1080.29 & 0.04 & 1334.1 & 0.01 \\
\hline 1138.4 & 0.01 & 1347.27 & 0.48 \\
\hline 1167.53 & 0.06 & 1384.01 & 0.01 \\
\hline 1174.42 & 0.02 & 1419.12 & 0.18 \\
\hline 1188.16 & 0.01 & 1430.17 & 0.17 \\
\hline 1201.92 & 0.04 & 1437.51 & 0.03 \\
\hline 1229.58 & 0.07 & 1446.48 & 0.21 \\
\hline 1260.16 & 0.12 & 1480.93 & 0.13 \\
\hline 1261.91 & 0.03 & 1503.48 & 0.06 \\
\hline 1286.46 & 0.02 & 1510.92 & 0.12 \\
\hline 1295.7 & 0.05 & 1522.75 & 0.20 \\
\hline 1319.37 & 0.01 & 1547.63 & 0.36 \\
\hline 1347.9 & 0.01 & 1568.01 & 0.02 \\
\hline 1383.03 & 0.11 & 1586.82 & 1.00 \\
\hline 1423.6 & 0.09 & 1608.12 & 0.10 \\
\hline
\end{tabular}

1-phenylphenanthrene

$\begin{array}{lccc}1439.08 & 0.09 & 3091.49 & 0.01 \\ 1441.83 & 0.11 & 3098.47 & 0.04 \\ 1461.13 & 0.25 & 3102.4 & 0.02 \\ 1493.23 & 0.11 & 3114.96 & 0.01\end{array}$

$1503.04 \quad 0.15$

$1530.44 \quad 0.11$

$1574.53 \quad 0.06$

$1578.47 \quad 0.06$

$1598.72 \quad 0.13$

$1608.28 \quad 0.13$

$1617.38 \quad 0.04$

$1627.14 \quad 0.01$

$3048.94 \quad 0.01$

$3049.81 \quad 0.08$

$3053.16 \quad 0.24$

$3056.08 \quad 0.04$

$3057.45 \quad 0.08$

$3059.81 \quad 0.49$

$3065.83 \quad 0.43$

$3071.05 \quad 0.53$

$3072.25 \quad 1.00$

$3075.27 \quad 0.31$

$3079.62 \quad 0.47$

$3083.95 \quad 0.22$

$3097.08 \quad 0.23$

$3099.2 \quad 0.63$

The relative intensities are obtained by normalizing with the strongest band of each set. Maximum absolute intensity: neutral, 1.12 Debye $^{2}$ /AMU- $\AA^{2}$; cation, 14.10 Debye $^{2} /$ AMU- $\AA^{2}$. 
Table 3: Infrared frequencies $\left(\mathrm{cm}^{-1}\right)$ and relative intensities for 2phenylphenanthrene

\begin{tabular}{|c|c|c|c|}
\hline \multicolumn{4}{|c|}{ 2-phenylphenanthrene } \\
\hline & Neutral & & Cation \\
\hline Frequency & Relative Intensity & Frequency & Relative Intensity \\
\hline 216.1 & 0.01 & 410.93 & 0.01 \\
\hline 226.99 & 0.04 & 443.15 & 0.01 \\
\hline 391.22 & 0.01 & 455.03 & 0.03 \\
\hline 430.49 & 0.11 & 464.61 & 0.01 \\
\hline 446.38 & 0.13 & 496.99 & 0.01 \\
\hline 459.55 & 0.01 & 538.3 & 0.01 \\
\hline 488.19 & 0.03 & 582.51 & 0.01 \\
\hline 513.61 & 0.05 & 659.69 & 0.02 \\
\hline 531.58 & 0.01 & 660.61 & 0.03 \\
\hline 535.71 & 0.05 & 698.15 & 0.02 \\
\hline 547.51 & 0.03 & 735.1 & 0.01 \\
\hline 629.01 & 0.01 & 743.74 & 0.02 \\
\hline 672.37 & 0.33 & 758.98 & 0.06 \\
\hline 692.97 & 0.49 & 796.76 & 0.03 \\
\hline 705.52 & 0.05 & 865.78 & 0.01 \\
\hline 713.91 & 0.06 & 869.08 & 0.02 \\
\hline 737.25 & 0.76 & 898.24 & 0.02 \\
\hline 744.66 & 0.28 & 978.32 & 0.07 \\
\hline 753.9 & 0.54 & 1006.24 & 0.03 \\
\hline 772.67 & 0.03 & 1029.72 & 0.01 \\
\hline 803.23 & 0.76 & 1088.06 & 0.06 \\
\hline 818.34 & 0.22 & 1119.08 & 0.15 \\
\hline 835.09 & 0.01 & 1155.04 & 0.02 \\
\hline 844.95 & 0.09 & 1163.38 & 0.01 \\
\hline 853.76 & 0.04 & 1165.2 & 0.01 \\
\hline 881.76 & 0.13 & 1192.86 & 0.12 \\
\hline 883.15 & 0.29 & 1197.72 & 0.01 \\
\hline 904.23 & 0.02 & 1205.79 & 0.01 \\
\hline 912.49 & 0.06 & 1238.88 & 0.04 \\
\hline 930.4 & 0.02 & 1253.2 & 0.01 \\
\hline 940.72 & 0.01 & 1272.58 & 0.04 \\
\hline 983.28 & 0.08 & 1305.26 & 0.02 \\
\hline 987.3 & 0.01 & 1312.25 & 0.09 \\
\hline 1020.05 & 0.09 & 1332.15 & 0.13 \\
\hline 1035.87 & 0.05 & 1343.27 & 0.01 \\
\hline 1045.8 & 0.01 & 1351.62 & 0.12 \\
\hline 1080.04 & 0.07 & 1398.92 & 0.03 \\
\hline 1094.55 & 0.01 & 1410.71 & 0.04 \\
\hline 1145.08 & 0.03 & 1420.15 & 0.06 \\
\hline 1162.84 & 0.01 & 1441.27 & 0.03 \\
\hline 1180.91 & 0.05 & 1449.27 & 0.07 \\
\hline 1186.33 & 0.07 & 1461.94 & 0.04 \\
\hline 1204.91 & 0.02 & 1494.1 & 0.07 \\
\hline 1237.93 & 0.15 & 1515.57 & 0.03 \\
\hline 1266.29 & 0.07 & 1583.36 & 1.00 \\
\hline 1286.68 & 0.05 & 1595.2 & 0.26 \\
\hline 1305.32 & 0.01 & 1599.03 & 0.02 \\
\hline 1325.84 & 0.01 & 1622.01 & 0.03 \\
\hline 1343.21 & 0.01 & 3088.69 & 0.01 \\
\hline 1345.87 & 0.01 & 3098.71 & 0.01 \\
\hline 1383.35 & 0.05 & 3101.52 & 0.02 \\
\hline 1421.77 & 0.06 & 3109.09 & 0.01 \\
\hline 1427.34 & 0.04 & & \\
\hline 1447.47 & 0.08 & & \\
\hline 1464.13 & 0.51 & & \\
\hline 1492.25 & 0.48 & & \\
\hline 1501.51 & 0.08 & & \\
\hline 1531.19 & 0.01 & & \\
\hline 1567.46 & 0.02 & & \\
\hline 1585.34 & 0.03 & & \\
\hline 1606.62 & 0.11 & & \\
\hline 1611.06 & 0.23 & & \\
\hline 1619.15 & 0.06 & & \\
\hline 1624.73 & 0.19 & & \\
\hline 3046.91 & 0.01 & & \\
\hline
\end{tabular}

$\begin{array}{cc}3049.17 & 0.11 \\ 3050.97 & 0.20 \\ 3055.62 & 0.18 \\ 3058.19 & 0.15 \\ 3058.9 & 0.21 \\ 3064.62 & 0.40 \\ 3066.47 & 0.76 \\ 3068.59 & 0.10 \\ 3072.92 & 1.00 \\ 3074.13 & 0.63 \\ 3080.58 & 0.08 \\ 3081.44 & 0.76 \\ 3092.97 & 0.67\end{array}$

The relative intensities are obtained by normalizing with the strongest band of each set. Maximum absolute intensity: neutral, 1.04 Debye ${ }^{2} / \mathrm{AMU}-\AA^{2}$; cation, 27.03 Debye ${ }^{2} / \mathrm{AMU}-\AA^{2}$. 
Int. J. Sci. Res. in Physics and Applied Sciences

Vol.6(5), Oct 2018, E-ISSN: 2348-3423

Table 4: Infrared frequencies $\left(\mathrm{cm}^{-1}\right)$ and relative intensities for

\begin{tabular}{|c|c|c|c|}
\hline \multicolumn{4}{|c|}{ 3-phenylphenanthrene } \\
\hline \multicolumn{2}{|r|}{ Neutral } & & \multirow{2}{*}{$\begin{array}{l}\text { Cation } \\
\text { Relative Intensity }\end{array}$} \\
\hline Frequency & Relative Intensity & Frequency & \\
\hline 106.26 & 0.01 & 262.31 & 0.01 \\
\hline 112.83 & 0.01 & 391.94 & 0.01 \\
\hline 217.78 & 0.01 & 411.89 & 0.01 \\
\hline 304.56 & 0.02 & 504.93 & 0.01 \\
\hline 365.47 & 0.02 & 562.74 & 0.02 \\
\hline 409.19 & 0.02 & 596.44 & 0.01 \\
\hline 416.52 & 0.02 & 607.84 & 0.01 \\
\hline 427.51 & 0.08 & 618.06 & 0.01 \\
\hline 453.77 & 0.03 & 667.52 & 0.02 \\
\hline 505.79 & 0.03 & 677.33 & 0.03 \\
\hline 512.93 & 0.09 & 706.69 & 0.02 \\
\hline 525.5 & 0.04 & 738.03 & 0.03 \\
\hline 552.72 & 0.03 & 746.76 & 0.04 \\
\hline 566.03 & 0.11 & 765.74 & 0.03 \\
\hline 611.25 & 0.02 & 820.57 & 0.01 \\
\hline 620.81 & 0.10 & 844.55 & 0.02 \\
\hline 626.59 & 0.07 & 850.75 & 0.03 \\
\hline 680.11 & 0.05 & 859.84 & 0.02 \\
\hline 692.95 & 0.42 & 870.97 & 0.02 \\
\hline 705.78 & 0.06 & 880.91 & 0.01 \\
\hline 709.41 & 0.09 & 978.38 & 0.06 \\
\hline 736.12 & 0.64 & 984.34 & 0.01 \\
\hline 738.51 & 0.29 & 1026.36 & 0.02 \\
\hline 755.59 & 0.42 & 1093.65 & 0.01 \\
\hline 777.27 & 0.01 & 1095.31 & 0.02 \\
\hline 794.49 & 0.21 & 1138.71 & 0.10 \\
\hline 834.87 & 0.02 & 1159.87 & 0.01 \\
\hline 837.38 & 0.87 & 1164.14 & 0.01 \\
\hline 848.64 & 0.05 & 1192.55 & 0.20 \\
\hline 856.34 & 0.24 & 1208.1 & 0.04 \\
\hline 865.03 & 0.11 & 1221.71 & 0.04 \\
\hline 869.79 & 0.06 & 1247.25 & 0.11 \\
\hline 903.26 & 0.02 & 1260.16 & 0.08 \\
\hline 917.34 & 0.06 & 1277.91 & 0.01 \\
\hline 934.3 & 0.01 & 1316.14 & 0.10 \\
\hline 940.48 & 0.01 & 1332.53 & 0.12 \\
\hline 985.84 & 0.01 & 1344.17 & 0.04 \\
\hline 991.61 & 0.06 & 1356.02 & 0.07 \\
\hline 1023.83 & 0.03 & 1408.59 & 0.10 \\
\hline 1034.71 & 0.10 & 1418.79 & 0.07 \\
\hline 1044.11 & 0.02 & 1443.98 & 0.03 \\
\hline 1078.89 & 0.07 & 1453.51 & 0.10 \\
\hline 1094.77 & 0.04 & 1476.23 & 0.01 \\
\hline 1146.01 & 0.03 & 1491.97 & 0.05 \\
\hline 1160.46 & 0.02 & 1524.57 & 0.21 \\
\hline 1180.15 & 0.03 & 1549.71 & 0.01 \\
\hline 1197.27 & 0.02 & 1556.02 & 1.00 \\
\hline 1214.56 & 0.01 & 1583.69 & 0.21 \\
\hline 1227.45 & 0.19 & 1596.86 & 0.05 \\
\hline 1250.07 & 0.07 & 1605.1 & 0.17 \\
\hline 1269.44 & 0.01 & 3089.01 & 0.01 \\
\hline 1280.59 & 0.05 & 3101.15 & 0.03 \\
\hline 1302.51 & 0.01 & 3101.71 & 0.01 \\
\hline 1325.08 & 0.02 & & \\
\hline 1340.14 & 0.01 & & \\
\hline 1349.38 & 0.04 & & \\
\hline 1396.19 & 0.10 & & \\
\hline 1421.77 & 0.03 & & \\
\hline 1426.28 & 0.02 & & \\
\hline 1448.49 & 0.12 & & \\
\hline 1454.08 & 0.28 & & \\
\hline 1496.6 & 0.45 & & \\
\hline 1511.56 & 0.15 & & \\
\hline 1519.11 & 0.05 & & \\
\hline 1565.62 & 0.02 & & \\
\hline
\end{tabular}

$\begin{array}{cc}\text { 3-phenylphenanthrene } \\ 1585.49 & 0.03 \\ 1609.25 & 0.07 \\ 1612.13 & 0.35 \\ 1618.28 & 0.08 \\ 1623.18 & 0.05 \\ 3047.38 & 0.01 \\ 3049.62 & 0.20 \\ 3050.92 & 0.13 \\ 3051.84 & 0.14 \\ 3057.02 & 0.08 \\ 3060.03 & 0.16 \\ 3066.36 & 0.62 \\ 3067.47 & 0.60 \\ 3071.31 & 0.40 \\ 3073.56 & 1.00 \\ 3075.74 & 0.53 \\ 3080.14 & 0.15 \\ 3081.07 & 0.59 \\ 3094.36 & 0.40\end{array}$

The relative intensities are obtained by normalizing with the strongest band of each set. Maximum absolute intensity: neutral, 1.11 Debye $^{2}$ AMU- $\AA^{2}$; cation, 21.68 Debye $^{2} /$ AMU- $\AA^{2}$. 
Table 5: Infrared frequencies $\left(\mathrm{cm}^{-1}\right)$ and relative intensities for 4-phenylphenanthrene

\begin{tabular}{|c|c|c|c|}
\hline \multicolumn{4}{|c|}{ 4-phenylphenanthrene } \\
\hline & Neutral & & Cation \\
\hline Frequency & Relative Intensity & Frequency & Relative Intensity \\
\hline 103.55 & 0.02 & 49.88 & 0.01 \\
\hline 198.3 & 0.01 & 126.36 & 0.04 \\
\hline 220.05 & 0.02 & 182.34 & 0.04 \\
\hline 230.13 & 0.02 & 260.38 & 0.02 \\
\hline 306.07 & 0.01 & 290.88 & 0.01 \\
\hline 329.2 & 0.01 & 330 & 0.04 \\
\hline 402.53 & 0.01 & 376.86 & 0.20 \\
\hline 405.43 & 0.01 & 448.43 & 0.03 \\
\hline 409.08 & 0.01 & 476.08 & 0.07 \\
\hline 455.42 & 0.01 & 486.5 & 0.01 \\
\hline 478.4 & 0.01 & 506.18 & 0.06 \\
\hline 494.84 & 0.02 & 532.69 & 0.03 \\
\hline 508 & 0.03 & 534.84 & 0.01 \\
\hline 535.67 & 0.13 & 654.66 & 0.07 \\
\hline 556.62 & 0.12 & 677.15 & 0.06 \\
\hline 605.27 & 0.05 & 695.53 & 0.02 \\
\hline 609.19 & 0.11 & 701.83 & 0.01 \\
\hline 625.71 & 0.01 & 745.34 & 0.06 \\
\hline 662.79 & 0.05 & 747.45 & 0.03 \\
\hline 695.77 & 0.55 & 756.31 & 0.06 \\
\hline 705.58 & 0.04 & 763.37 & 0.03 \\
\hline 713.46 & 0.21 & 792.66 & 0.01 \\
\hline 736.29 & 0.86 & 818.99 & 0.13 \\
\hline 757.62 & 0.32 & 828.48 & 0.06 \\
\hline 761.73 & 0.07 & 831.86 & 0.05 \\
\hline 767.48 & 0.09 & 864.31 & 0.04 \\
\hline 788.29 & 0.16 & 900.27 & 0.10 \\
\hline 821.82 & 0.67 & 931.33 & 0.01 \\
\hline 826.49 & 0.33 & 947.62 & 0.01 \\
\hline 857.59 & 0.11 & 959.83 & 0.01 \\
\hline 887.81 & 0.02 & 969.69 & 0.04 \\
\hline 899.07 & 0.03 & 976.06 & 0.01 \\
\hline 912.25 & 0.06 & 980.48 & 0.09 \\
\hline 924.91 & 0.04 & 1062.28 & 0.02 \\
\hline 936.43 & 0.02 & 1086.17 & 0.01 \\
\hline 958.7 & 0.02 & 1094.02 & 0.10 \\
\hline 980.86 & 0.04 & 1121.79 & 0.37 \\
\hline 985.35 & 0.02 & 1138.84 & 0.17 \\
\hline 1020.04 & 0.08 & 1180.84 & 0.02 \\
\hline
\end{tabular}

The relative intensities are obtained by normalizing with the strongest band of each set. Maximum absolute intensity: neutral, 1.08 Debye $^{2} / A M U-\AA^{2}$; cation, 12.82 Debye $^{2} /$ AMU- $\AA^{2}$.

$\begin{array}{llll}1040.33 & 0.04 & 1183.66 & 0.31 \\ 1064.92 & 0.04 & 1205.86 & 0.08 \\ 1071.19 & 0.06 & 1218.94 & 0.01 \\ 1087.53 & 0.04 & 1241.99 & 0.04 \\ 1135.03 & 0.05 & 1256.19 & 0.49 \\ 1160.46 & 0.10 & 1293.99 & 0.02 \\ 1171.76 & 0.01 & 1305.68 & 0.15 \\ 1175.03 & 0.03 & 1326.95 & 0.30 \\ 1199.59 & 0.02 & 1337.81 & 0.11 \\ 1212.96 & 0.03 & 1343.35 & 0.03 \\ 1248.02 & 0.05 & 1380.65 & 0.03 \\ 1260.47 & 0.06 & 1399.54 & 1.00 \\ 1282.94 & 0.03 & 1420.5 & 0.01 \\ 1291.56 & 0.13 & 1444.5 & 0.15 \\ 1312.41 & 0.02 & 1450.48 & 0.06 \\ 1318.69 & 0.02 & 1483.95 & 0.02 \\ 1383.75 & 0.09 & 1498.31 & 0.07 \\ 1435.11 & 0.21 & 1519.86 & 0.07 \\ 1437.91 & 0.12 & 1540.92 & 0.38 \\ 1448.96 & 0.20 & 1548.08 & 0.01 \\ 1491.26 & 0.20 & 1565.16 & 0.59 \\ 1498.04 & 0.03 & 1591.73 & 0.23 \\ 1526.71 & 0.03 & 1603.69 & 0.05 \\ 1569.24 & 0.04 & 3083.52 & 0.01 \\ 1579.61 & 0.05 & 3089.56 & 0.01 \\ 1595.93 & 0.04 & 3091.38 & 0.01 \\ 1605.17 & 0.14 & 3093.95 & 0.01 \\ 1615.2 & 0.05 & 3098.75 & 0.03 \\ 1628.2 & 0.02 & 3099.94 & 0.03 \\ 3046.37 & 0.02 & 3123.2 & 0.01 \\ 3048.92 & 0.09 & & \end{array}$

31

08

0.04

0.49

.02

0.11

.03

0.01

02

0.07

0.07

0.38

0.59

0.23

0.05

0.01

0.01

.01

0.03

0.01 
Int. J. Sci. Res. in Physics and Applied Sciences

Vol.6(5), Oct 2018, E-ISSN: 2348-3423

Table 6: Infrared frequencies $\left(\mathrm{cm}^{-1}\right)$ and relative intensities for 9-

\begin{tabular}{cccc}
\hline \multicolumn{4}{c}{ 9-phenylphenanthrene } \\
& Neutral & & Cation \\
\hline Frequency & Relative Intensity & Frequency & Relative Intensity \\
\hline 103.21 & 0.01 & 233.15 & 0.02 \\
202.33 & 0.01 & 259.32 & 0.01 \\
237.29 & 0.01 & 320.87 & 0.01 \\
256.55 & 0.01 & 389.08 & 0.03 \\
277.14 & 0.02 & 399.91 & 0.02 \\
401.7 & 0.03 & 422.33 & 0.01 \\
406.15 & 0.01 & 475.84 & 0.01 \\
427.07 & 0.07 & 535.06 & 0.01 \\
455.19 & 0.01 & 560.45 & 0.08 \\
479.14 & 0.02 & 573.36 & 0.02 \\
510.01 & 0.05 & 597.05 & 0.02 \\
559.34 & 0.02 & 601.79 & 0.01 \\
563.73 & 0.14 & 653.02 & 0.02 \\
582.42 & 0.13 & 681.26 & 0.07 \\
609.89 & 0.01 & 698.4 & 0.04 \\
613.76 & 0.15 & 704.31 & 0.07 \\
634.71 & 0.03 & 745.09 & 0.01 \\
657.59 & 0.06 & 750.3 & 0.03 \\
695.25 & 0.63 & 766.45 & 0.08 \\
704.53 & 0.07 & 772.38 & 0.13 \\
719.88 & 0.49 & 831.19 & 0.04 \\
738.35 & 0.74 & 845.86 & 0.02 \\
745.2 & 0.15 & 858.63 & 0.01 \\
758.74 & 0.56 & 904.93 & 0.04 \\
769.15 & 0.60 & 915.59 & 0.05 \\
779.71 & 0.03 & 933.76 & 0.01 \\
837.24 & 0.01 & 954.26 & 0.01 \\
844.3 & 0.03 & 961.97 & 0.01 \\
890.49 & 0.29 & 979.81 & 0.05 \\
904.85 & 0.05 & 981.35 & 0.03 \\
912.89 & 0.05 & 983.19 & 0.01 \\
925.88 & 0.07 & 1018.55 & 0.01 \\
927.66 & 0.08 & 1031.35 & 0.01 \\
941.93 & 0.01 & 1048.69 & 0.01 \\
986.18 & 0.05 & 1087.2 & 0.01 \\
986.64 & 0.01 & 1095.48 & 0.01 \\
1026.47 & 0.08 & 1129.61 & 0.09 \\
1033.42 & 0.08 & 1158.19 & 0.09 \\
\hline
\end{tabular}

phenylphenanthrene

1043.79

1076.01

0.10

1132.89

1150.59

1158.67

1167.17

1176.89

1205.46

1230.3

1241.7

1287.73

1305.89

1322.56

1376.6

1422.16

1441.13

1443.88

1451.6

1492.93

1496.5

1533.79

1575.79

1582.06

1604.57

1609.76

1618.99

3049.12

3051.31

3056.06

3056.81

3058.57

3060.66

3067.77

3069.9

3073.58

3074.89

3080.68

3081.77

3094.14

0.09

1184.3

1211.52

1235.2

1265.09

1278.29

1290.09

1315.85

1326.97

1334.41

1353.73

1368.15

1426.71

1430.84

1440.55

1445.19

1469.16

1481.77

1530.7

1547.97

1560.87

1572.89

1589.14

1603.57

3093.31

3099.73

3103.02

3113.49

0.02

0.20

0.04

0.13

0.12

0.02

0.06

0.15

0.17

0.03

0.43

0.31

0.02

0.04

0.06

0.28

0.04

0.03

0.02

0.04

0.01

0.89

0.04

0.17
0.08

0.06

0.04

0.08

0.16

0.07

0.07

0.45

0.53

1.00

0.72

0.33

0.50

0.36

3096.26

0.83

0.45

The relative intensities are obtained by normalizing with the strongest band of each set. Maximum absolute intensity: neutral, 0.97 Debye $\mathrm{D}^{2} / \mathrm{AMU}-\AA^{2}$; cation, 8.59 Debye $^{2} / \mathrm{AMU}-\AA^{2}$. 